\title{
Desmoplastic Infantile Ganglioglioma
}

National Cancer Institute

\section{Source}

National Cancer Institute. Desmoplastic Infantile Ganglioglioma. NCI Thesaurus. Code C4738.

A WHO grade I large cystic tumor that occurs almost exclusively in infants, with a prominent desmoplastic stroma having a neuroepithelial population of neoplastic astrocytes together with a variable neuronal component. It involves the superficial cerebral cortex and leptomeninges, and often attaches to the dura. Although clinically it presents as a large tumor, it generally has a good prognosis following surgical resection. (Adapted from WHO) 